\title{
Feminización de juveniles del robalo blanco Centropomus undecimalis (Bloch 1792) usando 17ß-estradiol
}

\section{Feminization of young common Snook Centropomus undecimalis (Bloch 1792) using 17ß-estradiol}

\author{
Juan Manuel Vidal-López ${ }^{1}$, Carlos Alfonso Álvarez-González ${ }^{1 *}$, Wilfrido Miguel Contreras- \\ Sánchez ${ }^{1}$, Reynaldo Patiño ${ }^{2}$, Arlette Amalia Hernández-Franyutti ${ }^{1}$, Ulises Hernández-Vidal ${ }^{1}$ y \\ Rafael Martínez García
}

\begin{abstract}
RESUMEN
El robalo blanco Centropomus undecimalis es un pez protándrico hermafrodita con un alto valor comercial y potencial de cultivo en México y Estados Unidos de América, y en Centroamérica y el Caribe es sustento importante de su pesca artesanal. Diversos estudios indican que las hembras aparentemente presentan un mayor crecimiento que los machos. El objetivo del presente estudio consistió en evaluar el efecto de la suplementación del esteroide $17 \beta$-estradiol (E2) en el alimento sobre la proporción de sexos de esta especie bajo condiciones de laboratorio. Para ello se realizó un estudio donde se evaluaron seis tratamientos (tiempos de exposición) con $17 \beta$-estradiol $(7,14,21,28,35$ y 42 días) usando alimento impregnado con $50 \mathrm{mg} \mathrm{kg}^{-1}$ de $17 \beta$-estradiol y una dieta control sin esteroide. Después de los días de exposición, los peces fueron mantenidos con dieta sin 17ß-estradiol hasta completar 204 días de crianza, determinándose la proporción de sexos, crecimiento y supervivencia. Al final del período de crianza, los peces alimentados con dieta enriquecida con $17 \beta$-estradiol por 21 y hasta 42 días muestran la mayor proporción de hembras (93\%), mientras que el grupo control presentó el $100 \%$ de machos. El mayor crecimiento en longitud y peso se obtuvo en los peces alimentados por 21 días con el E2 (193.11 $\pm 1.83 \mathrm{~mm}$ y $28.56 \pm 0.63 \mathrm{~g})$ comparado con el resto. La supervivencia no mostró diferencias significativas entre los tratamientos (92\% a 98\%). Se concluye que es posible obtener altos porcentajes de feminización utilizando $17 \beta$-estradiol al alimentar a los peces por al menos 21 días.
\end{abstract}

Palabras claves: Feminización, 17ß-estradiol, robalo blanco.

\begin{abstract}
Common snook Centropomus undecimalis is a protandric fish with a high commercial importance and aquacultural potential in Mexico and the United States. Several studies indicate that females have higher growth capacity than males. For this reason, the objective of this research was to evaluate the effect of a $17 \beta$-estradiol (E2) diet supplementation on sex proportion for this species. In this sense, an experimental study was conducted where fish were fed for different time periods $(7,14,21,28,35$, and 42 days) with food impregnated with $50 \mathrm{mg}$ of $\mathrm{E} 2 / \mathrm{Kg}$, and one control diet without the presence of the steroid. After feeding times, fish were raised for additional 204 days with the control diet to evaluate sex proportion, growth and survival. Our results showed that fish fed with E2 for 21 days or more had the highest female sex proportion (93-100\%), while the control group showed the highest male proportion $(100 \%)$. The highest growth (weight and total length) was detected in fish fed with E2 for 21 days (193.11 $\pm 1.83 \mathrm{~mm}$ and 28.56

1 Laboratorio de Acuicultura Tropical, División Académica de Ciencias Biológicas, Universidad Juárez Autónoma de Tabasco, Carretera Villahermosa-Cárdenas Km 0.5, C.P. 86039, Villahermosa, Tabasco, México, TeleFax: 52(993) 1614591.alvarez_alfonso@hotmail.com*

2 USGS Texas Cooperative Fish and Wildlife Research Unit, 15th and Boston, Agricultural Sciences Rm 218. Lubbock, TX 79409-2120. Phone 806-742-285, Fax 806-742-294.
\end{abstract}

Recibido 5-VII-2011

Aceptado 7-VIII-2012

DOI: http://dx.doi.org/10.15359/revmar.4.5 
$\pm 0.63 \mathrm{~g})$ compared with the rest of the treatments. Survival did not show statistical differences between treatments $(92-98 \%)$. We conclude that high percentage of $C$. undecimalis females can be obtained when fish are fed for 21 days or more with artificial food supplemented with E2.

Keywords: Feminization, 17ß-estradiol, common snook.

\section{INTRODUCCIÓN}

El robalo blanco Centropomus undecimalis se ubica como una de las especies con mayor importancia económica para el Golfo de México, además de tener un alto potencial de cultivo (Chapman et al. 1982; Sánchez et al. 2002; ÁlvarezLajonchére, 2004). Sin embargo, aún existen diversas dudas en relación con su biología y ecología. Una duda importante tiene que ver con su crecimiento, ya que es considerada una especie protándrica hermafrodita que madura sexualmente primero como macho y luego cambia a hembra (Caballero, 1996; Taylor et al. 2000), pasando la mayor parte de su ciclo de vida en ambientes dulceacuícolas y cuando son adultos migran periódicamente al medio marino para la temporada reproductiva (Caballero, 1996; Tringali y Bert, 1996; Peters et al. 1998; PereraGarcía et al. 2011). Desde el punto de vista de su cultivo, se ha determinado que tiene un crecimiento rápido, se adapta con facilidad al manejo y a diferentes condiciones medioambientales, presenta una apariencia física atractiva, además de ser una especie muy apreciada por su valor económico (Tucker, 1987; Taylor et al. 2000; Sánchez et al. 2002; Tucker, 2003). Taylor et al. (2000) reportaron un crecimiento diferencial entre hembras y machos, mencionando que las hembras presentan un crecimiento mayor al de los machos. En este aspecto, se considera que esta diferencia de crecimiento se debe a su condición protándrico-hermafrodita, al cambiar de macho a hembra. Esta condición podría presentar ventajas en el cultivo de la especie; por lo que se debe considerar la posibilidad de incluir poblaciones monosexo (100\% hembras). En acuicultura, el uso de esteroides sexuales, tales como los andrógenos (17a-metiltestosterona, ketotestosterona y dihidrotestosterona) y los estrógenos (17 $\beta$-estradiol y estrona) juegan un papel importante en la manipulación de la diferenciación de las gónadas (Yamamoto, 1969). De esta manera, los métodos que comúnmente se emplean para modificar la diferenciación del sexo involucran realizar mezclas de los esteroides con alimento artificial, inmersión de crías en el esteroide diluido en agua, administrarlo en los alimentos vivos (nauplios de Artemia spp.) como vehículo (Piferrer, 2001; Stewart et al. 2001; Devlin y Nagahama, 2002; Contreras-Sánchez et al. 2003; Vidal-López et al. 2009).

Para llevar a cabo la feminización se ha utilizado ampliamente el $17 \beta$-estradiol (E2), esto ha sido reportado en el pargo de cabeza negra Acanthopagrus schlegeli, un pez protogínico, donde el uso de $17 \beta$-estradiol en machos de dos años de edad aplicado en el alimento durante la época reproductiva permitió obtener hasta un $100 \%$ de hembras (Chang y Lee, 1992). Strüssmann et al. (1996) obtuvieron el $100 \%$ de hembras del pejerrey $O$. bonariensis, utilizando alimento impregnado con $17 \beta$-estradiol a dosis de 20 y $50 \mathrm{mg} \mathrm{kg}^{-1}$ de alimento. ContrerasSánchez et al. (2003) y Vázquez (2008) reportaron porcentajes de 60 y $100 \%$ de 
hembras empleando dosis de 50 y $100 \mathrm{mg}$ E2 $\mathrm{kg}^{-1}$ en el alimento usado en larvas del pejelagarto Atractosteus tropicus. De esta forma, el objetivo de la presente investigación fue evaluar el efecto del esteroide $17 \beta$-estradiol en la proporción de sexos en juveniles de $C$. undecimalis.

\section{MATERIALES Y MÉTODOS}

\section{Recolecta, aclimatación e identificación de especímenes}

Se capturaron 650 juveniles de robalo blanco C. undecimalis en la laguna costera de Mecoacán $\left(18^{\circ} 24.35^{\prime} \mathrm{N}\right.$ y $93^{\circ} 04.46^{\prime}$ W, GPS $60 \mathrm{C}_{\mathrm{x}}$, Garmin, Taiwán) en el mes de julio de 2010, en el municipio de Paraíso, Tabasco, México. La captura se realizó con red de arrastre de $15 \mathrm{~m}$ de longitud y $3 \mathrm{~m}$ de altura, elaborada con malla plástica tipo mosquitero. Los peces fueron transportados a las instalaciones del Laboratorio de Acuicultura Tropical de la DACBIOL-UJAT, donde fueron aclimatados durante una semana, previo al inicio de la fase experimental. Los especímenes fueron identificados usando los criterios generales de Rivas (1986), tales como, longitud de la espina caudal, número de radios en la aleta dorsal y caudal, altura del cuerpo, y al final, antes de realizar el procedimiento histológico, se corroboró mediante la reidentificación si pertenecían a la especie deseada.

\section{Diseño experimental}

El experimento se realizó en un sistema de recirculación cerrado, compuesto de 21 tanques cilindrocónicos de fibra de vidrio de $70 \mathrm{~L}$ de capacidad. El sistema fue integrado de una bomba de succión de 3/4 Hp (Jacuzzi ${ }^{\circledR}$, Toronto, Ontario, Canadá), filtro de arena, filtro sedimentador y reservorio de $500 \mathrm{~L}$. El experimento consistió en seis tratamientos correspondientes a tiempos o días de exposición durante los que fue suministrado el alimento impregnado con $17 \beta$-estradiol $\left(50 \mathrm{mg} \mathrm{kg}^{-1} \mathrm{de}\right.$ alimento) por 7, 14, 21, 28, 35 y 42 días, además de incluirse un grupo control, el cual no fue impregnado con el esteroide y solo fue mezclado con alcohol etílico (usado como vehículo). Todos los tratamientos fueron evaluados por triplicado y en cada réplica se sembraron 20 juveniles con talla y peso inicial de $46.89 \pm 8.61 \mathrm{~mm}$ y $2.05 \pm$ $0.71 \mathrm{~g}$, respectivamente.

\section{Alimentación de los especímenes}

Los especímenes fueron alimentados usando una estrategia de sustitución parcial o coalimentación a base de Artemia: nauplios de Artemia spp. (450 nauplios/ ml) desde el primero hasta el décimo día una vez sembrados en el sistema, biomasa de Artemia spp. del día 5 al 15 (200 g de biomasa de Artemia spp. congelada al inicio con un aumento diario de 5 a 8 g) y, finalmente, con alimento balanceado para trucha (Silver Cup, $45 \%$ proteína y $16 \%$ lípidos) a partir del día 12 hasta lograr la total adaptación al consumo de alimento artificial. La aplicación del E2, usando los nauplios de Artemia spp. como vehículo e incorporar el esteroide en la biomasa de Artemia spp., se realizó de acuerdo con la metodología señalada por Stewart et al. (2001), empleando una dosis de $50 \mathrm{mg} \mathrm{E} 2 \mathrm{~kg}^{-1}$ de medio de incubación o de alimento. Para incorporar el alimento balanceado, se siguió la metodología de preparación reportada por Pandian y Sheela (1995); Green et al. (1997) y Phelps et al. (1996), usando la misma dosis que en la Artemia. Para la preparación del alimento control, se utilizó solamente el alcohol etílico (vehículo) al $96 \%$ en el que se disolvió el esteroide. 
La alimentación se realizó 5 veces al día (7:00, 10:00, 13:00, 15:00 y 18:00) (Contreras-Sánchez et al. 2003).

\section{Calidad del agua}

Diariamente, se midieron el oxígeno disuelto, la temperatura, el $\mathrm{pH}$ y la salinidad. El oxígeno disuelto (7.02 \pm 0.02 $\mathrm{mg} \mathrm{L}^{-1}$ ) se midió con un oxímetro (YSI 55, California, USA), la temperatura del agua se midió con un termómetro (YSI ${ }^{\circledR}$ 55, California, USA), registrándose valores promedio de $30.2^{\circ} \mathrm{C} \pm 0.14$. El pH (6.5 $\pm 0.07)$ fue medido con un potenciómetro (Denver Instrument UB-10, Denver, Colorado, USA), la salinidad (10 \pm 0.5 UPS) se midió con un refractómetro (Vee Gee ${ }^{\circledR}$, A366ATC, USA). Semanalmente, se midieron, con un equipo multiparamétrico (Hanna Instruments, HI 98311, Rhode Island, USA), las concentraciones de amonio $\left(0.1 \pm 0.01 \mathrm{mg} \mathrm{L}^{-1}\right)$, nitritos $(0.01 \pm$ $\left.0.01 \mathrm{mg} \mathrm{L}^{-1}\right)$ y nitratos $\left(0.5 \pm 0.03 \mathrm{mg} \mathrm{L}^{-1}\right)$.

\section{Evaluación del crecimiento y análisis histológico}

Una vez terminados los períodos experimentales, los peces fueron reubicados a un sistema abierto de 18 tanques cilíndricos de 2000 L de capacidad para realizar un crecimiento adicional por otros 204 días, manteniendo las mismas condiciones experimentales a partir de recambios totales semanales. Al final de este tiempo, se determinaron el peso con una balanza analítica (Ohaus ${ }^{\circledR}$, Pine Brook, NJ, EE. UU.) con precisión de $0.001 \mathrm{~g}$ y la longitud total con un vernier digital (Carbon Fiber Composites Digital ${ }^{\circledR}$ Atlanta, Georgia, USA) con precisión de $0.1 \mathrm{~mm}$. Las biometrías se obtuvieron de todos los organismos de cada tratamiento. Para determinar la proporción de sexos se tomó una muestra aleatoria de cinco peces de cada réplica al final de cada tratamiento (42 días) y del período de crianza (204 días). Los peces seleccionados fueron sacrificados con sobredosis de metansulfonato de tricaína (MS-222) a razón de 1 g/L. Posteriormente, fueron disectados y se extrajeron las gónadas de cada organismo (Fig. 2 a y b), las cuales fueron fijadas por $24 \mathrm{~h}$ en solución de Bouin y luego mantenidas en alcohol etílico al 70\%. Para el procesamiento

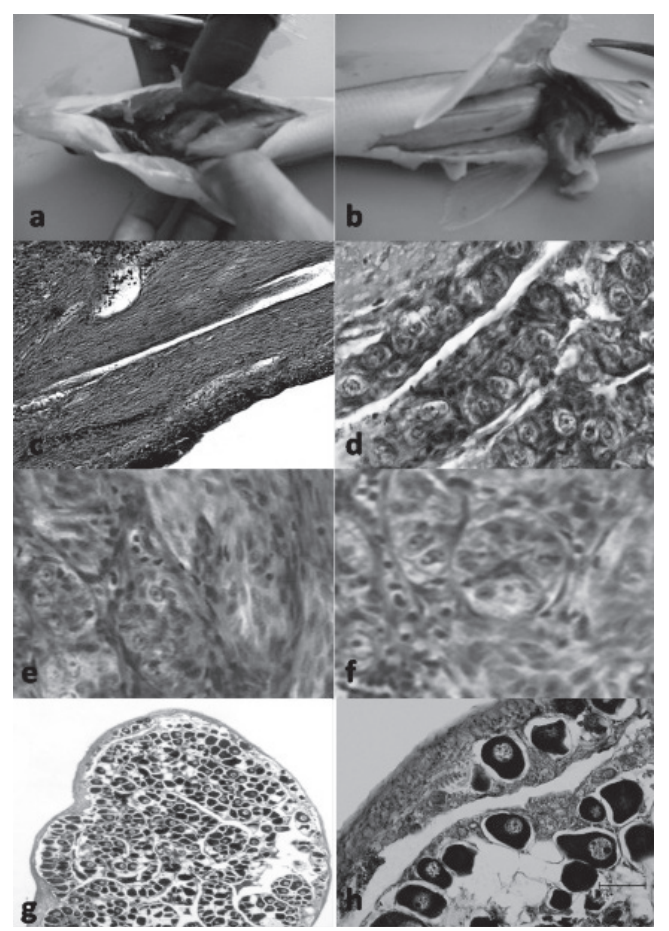

Fig. 2. Ubicación morfológica y corte transversal de gónadas de hembras (a) y machos (b) de C. undecimalis. Gónada indiferenciada (c $\mathrm{y} \mathrm{d}$, hematoxilina-eosina (H-E), 50 y $400 \mathrm{X}$ ). Testículo (e y f, H-E, 400 X). Ovario (g y h, Tricrómica de Masson, 50 y 400 X)

Fig. 2. Morphologic location and transversal cut of gonads from $C$. undecimalis females (a) and males (b). Undifferentiated gonad (c and d, hematoxilin-eosin (H-E), 50 and $400 \mathrm{X}$ ). Testicle (e and f, H-E, 400 $\mathrm{X}$ ). Ovary (g and h, Masson's trichrome stain, 50 and $400 \mathrm{X}$ ) 
de las muestras se siguió la técnica histológica convencional con tinción, basada en hematoxilina-eosina (H-E) (Aguilar et al. 1996), que consiste en deshidratación de las muestras con alcoholes sucesivos (70 a 100\%), aclaramiento con Xilol, inclusión en parafina, obtención de cortes de 6 y $7 \mu \mathrm{m}$, tinción con H-E y montaje de las muestras en bálsamo de Canadá. Para analizar las laminillas con las estructuras gonádicas se empleó un microscopio óptico (Zeiss ${ }^{\circledR}$, Göttingen, Germany) con los objetivos 10X, 40X, y las diferencias entre hembras y machos se basaron en los criterios reportados para la especie por Grier y Taylor (1998); Grier (2000) y Huber (2007). Al final del período de alimentación con el esteroide (42 días), el 100\% de los organismos muestreados se encontraban sexualmente indiferenciados en todos los tratamientos con 17 $\beta$-estradiol y el grupo control, esto se corroboró con análisis histológico. Debido a esta situación, se realizó una crianza adicional hasta completar los 204 días y lograr la diferenciación gonadal. Para este tiempo fue posible observar organismos diferenciados en machos y hembras.

\section{Análisis estadístico}

Para determinar si existían diferencias en crecimiento (longitud total y peso) entre los diferentes tratamientos, se aplicó un análisis de varianza (ANOVA) de una vía, empleándose la prueba a posteriori de Bonferroni, previo a la comprobación de la normalidad (prueba de Komogorov-Smirnov) y homogeneidad de varianza (prueba de Levine). Para comparar la proporción de sexos y la supervivencia entre los tratamientos (transformación Arco seno), se emplearon tablas de contingencia para la distribución $\chi^{2}$. Todos los análisis estadísticos fueron realizados con el programa
Statgraphics plus ${ }^{\circledR}$ versión 5.1 (StatPoint, 2006) con un valor de confianza del $95 \%$.

\section{RESULTADOS}

El análisis histológico de gónadas permitió detectar diferencias significativas en el porcentaje de hembras obtenidas en los tratamientos $\left(\chi^{2} ; P<0.05\right)$; en el grupo control se obtuvo un $100 \%$ de machos, mientras que los peces alimentados con el E2 presentaron variación desde un 13 hasta el 93\% de hembras (Fig. $2 \mathrm{~g} \mathrm{y} \mathrm{h}$ ). Los peces tratados de 7 a 14 días con $17 \beta$-estradiol presentaron 13 y $15 \%$ de hembras, respectivamente, siendo diferentes estadísticamente en comparación con los peces alimentados con esteroide por $21,28,35$ y 42 días, obteniéndose valores desde 87 y $93 \%$, siendo estadísticamente iguales entre ellos (Fig. 1).

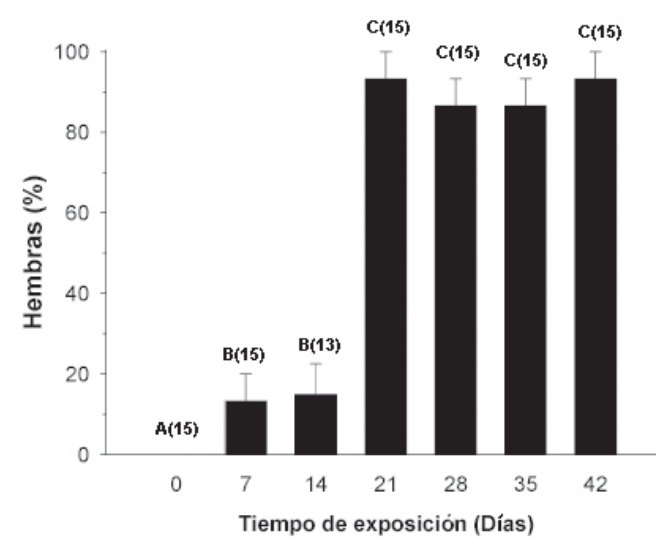

Fig. 1. Promedio ( \pm Error Estándar) del porcentaje de hembras de $C$. undecimalis tratadas con E2 después de un período de 246 días de crecimiento. Las letras iguales indican tratamientos que no fueron estadísticamente diferentes entre sí $\left(X^{2}, P>0.05\right)$. El asterisco $(*)$ indica el tamaño de muestra por tratamiento

Fig. 1. Average percentage ( \pm standard error) of C. undecimalis females treated with E2 after 246 days of growth. The same letters indicate equal statistical treatments $\left(X^{2}, P>0.05\right)$. Asterisk $(*)$ indicates the sample size per treatment 
La longitud total y el peso final a los 204 días mostraron diferencias significativas entre los tratamientos (ANOVA, $P<$ 0.05). La mayor longitud total (193.11 \pm $1.83 \mathrm{~mm})$ y peso $(28.56 \pm 0.63 \mathrm{~g}) \mathrm{se}$ obtuvieron a los 21 días de administración de E2. Los peces que menos crecieron en longitud $(170.04 \pm 2.17 \mathrm{~mm})$ fueron los de 35 días de tratamiento con E2, y en peso los del tratamiento a 42 días $(19.27 \pm 0.22$ g). La supervivencia no mostró diferencias entre los tratamientos $\left(\chi^{2} ; P>0.05\right)$, estando entre $92 \%$ y $98 \%$ (Cuadro 1).

\section{DISCUSIÓN}

Al finalizar el período de alimentación con el esteroide (42 días), los organismos presentaron una longitud total promedio de $81.84 \pm 2.9 \mathrm{~mm}$ y un peso promedio de $3.66 \pm 0.65 \mathrm{~g}$; para esta talla y tiempo máximo de alimentación con los tratamientos no fue posible identificar el sexo de los organismos, debido a que el $100 \%$ de los organismos muestreados presentaron gónadas en estado indiferenciado. A partir de los 204 días (período de crianza), los resultados indican que al suministrar el esteroide $17 \beta$-estradiol en el alimento por 21 días, se obtienen porcentajes altos de hembras. En este aspecto, resultados similares han sido reportados por Strüssmann et al. (1996), donde utilizando alimento impregnado del esteroide $17 \beta$-estradiol a dosis de 20 y $50 \mathrm{mg}$ $\mathrm{kg}^{-1}$ de alimento desde 105 hasta 179 días lograron obtener el $100 \%$ de hembras en el pejerrey $O$. bonariensis. Otros reportes como los de Goudie et al. (1983) con bagre de canal Ictalurus punctatus, Johnstone et al. (1978) con O. mykiss, Nakamura (1984) con O. keta y Colombo y Grandi (1990) con Anguila anguila, reafirman que el uso de $17 \beta$-estradiol para producir poblaciones monosexo en peces es efectivo. Asimismo, en otros trabajos con especies hermafroditas como la dorada Sparus aurata y el pargo de cabeza negra Acanthopagrus schlegeli, se ha observado que es posible inducir la inversión del sexo, obteniéndose porcentajes de hasta el $100 \%$ de hembras utilizando estradiol en la dieta a razón de $4 \mathrm{mg} \mathrm{kg}^{-1}$ de alimento por cinco meses de tratamiento para el caso del pargo cabeza negra (Happe y Zohar, 1988; Chang y Lee, 1992).

Cuadro 1. Crecimiento y supervivencia de juveniles de C. undecimalis alimentados por 42 días tratados con $\mathrm{E} 2$

Table 1. Growth and survival of C. undecimalis juveniles treated with E2 for 42 days

\begin{tabular}{cccc}
\hline \multirow{2}{*}{ Días } & Peso $(\mathbf{g})$ & Longitud total $(\mathbf{m m})$ & Supervivencia \\
\cline { 2 - 4 } & Promedio $\pm \mathbf{E E}$ & Promedio $\pm \mathbf{E E}$ & $(\mathbf{N})(\mathbf{\%})$ \\
\hline Control & $28.18 \pm 0.81 \mathrm{a}$ & $184.48 \pm 3.08 \mathrm{a}$ & $(58) 97$ \\
7 & $28.28 \pm 0.72 \mathrm{a}$ & $183.12 \pm 2.40 \mathrm{a}$ & $(57) 95$ \\
14 & $28.11 \pm 0.36 \mathrm{a}$ & $183.75 \pm 2.29 \mathrm{a}$ & $(56) 93$ \\
21 & $28.56 \pm 0.63 \mathrm{a}$ & $193.11 \pm 1.83 \mathrm{a}$ & $(56) 93$ \\
28 & $26.56 \pm 1.10 \mathrm{~b}$ & $171.80 \pm 3.06 \mathrm{~b}$ & $(55) 92$ \\
35 & $20.78 \pm 0.96 \mathrm{c}$ & $170.04 \pm 2.17 \mathrm{c}$ & $(59) 98$ \\
42 & $19.27 \pm 0.22 \mathrm{c}$ & $170.88 \pm 2.24 \mathrm{c}$ & $(59) 98$
\end{tabular}

$\mathrm{N}^{*}=$ número de organismos vivos. Las letras diferentes indican diferencias estadísticas entre el control y los tratamientos. 
Un criterio importante para inducir la inversión sexual mediante la administración oral de esteroides sintéticos, es identificar el período lábil en que las crías pueden ser susceptibles a un posible efecto de la hormona. En el caso de C. undecimalis se sabe que organismos juveniles con tallas aproximadas de $80 \mathrm{~mm}$ y edad aproximada de 67 días, aún no se han diferenciado (Contreras et al. 2007). En este aspecto, Hunter y Donaldson (1983) reportan que para identificar el período lábil de una especie se requiere de la aplicación del esteroide a distintos tiempos y usando diferentes dosis, es fundamental para asegurar una alta proporción de organismos monosexo. Sin embargo, en este estudio no se realizó la evaluación de diferentes dosis utilizando una dosis fija $\left(50 \mathrm{mg} \mathrm{kg}^{-1}\right.$ de alimento), obteniéndose altos porcentajes de feminización a partir de los 21 días de suministración de E2 sin detectar efectos adversos sobre la supervivencia de los organismos. Por otra parte, un suministro más corto del esteroide muestra una mayor proporción de machos, al grado que en la dieta control se obtuvieron organismos $100 \%$ machos. Esto indica que el proceso de inversión sexual en esta especie es también gobernado por el tiempo de exposición al esteroide, como ha sido documentado en otras especies como Oreochromis niloticus. En este aspecto, diversos autores han mencionado que la eficiencia de la inversión sexual con base en los tiempos de exposición al esteroide varía de acuerdo con la especie (Hunter y Donaldson, 1983; Phelps et al. 1996), por lo que para las crías de tilapia del Nilo, Oreochromis niloticus, el tiempo óptimo para obtener poblaciones monosexo $(100 \%$ machos con 17 a-metiltestosterona) se alcanza entre los 20 y los 28 días de exposición, planteándose que la diferenciación gonadal ocurre entre los 11 y 21 días poseclosión en organismos menores de $14 \mathrm{~mm}$ (Phelps et al. 1996; Green et al. 1997). Asimismo, Strüssmann et al. (1996) reportan que en el pejerrey $O$. bonariensis, el período lábil de feminización ocurre entre los 28 y 105 días, mientras que la diferenciación a ovario se lleva a cabo entre los 49 y 63 días a una talla de $18.2 \mathrm{~mm}$. Para C. undecimalis, la talla de diferenciación temprana a testículo se presenta a partir de los 110 $\mathrm{mm}$ y aproximadamente a 92 días de edad (Contreras et al. 2007). Por otra parte, el inicio del proceso de reversión sexual hacia hembra en esta especie se ha reportado que ocurre entre los 630 y $718 \mathrm{~mm}$, aunque esto fue determinado para juveniles silvestres, donde la edad de los peces no se conoce (Marshall, 1958); estos datos coinciden con los reportados por Taylor et al. (2000), quienes sugieren que la reversión hacia hembras ocurre entre los 600 a $700 \mathrm{~mm}$ y una edad de entre 5 y 7 años. Por otro lado, el análisis histológico en este estudio muestra que en las tallas entre 170 y $193 \mathrm{~mm}$ es posible observar hembras de C. undecimalis. Sin embargo, la edad de los organismos en esta investigación tampoco fue determinada al ser juveniles obtenidos del ambiente natural.

De esta manera, los datos de crecimiento en juveniles de $C$. undecimalis para este estudio muestran resultados prometedores, debido a que los valores de crecimiento en longitud y peso obtenidos son similares a los reportados por Sánchez et al. (2002) y Tucker (2003). Otro estudio realizado por Zarza et al. (2006) muestra datos de crecimientos en juveniles de $C$. undecimalis de $26.43 \pm 0.13 \mathrm{~cm}$ y $265.3 \pm$ $0.62 \mathrm{~g}$ en períodos de cultivo más prolongados. No obstante, en esta investigación se observan variaciones en crecimiento cuando los juveniles fueron alimentados 
con dieta que contenía el $17 \beta$-estradiol; por períodos más prolongados, el crecimiento fue menor a los peces del grupo control; de esta forma, resultados similares fueron obtenidos por ContrerasSánchez et al. (2003) y Vázquez (2008) en crías de pejelagarto, ambos estudios muestran un menor crecimiento en los tratamientos con el esteroide, además de una baja supervivencia, lo cual ha sido explicado por Flynn y Benfey (2007), quienes reportan que el uso del esteroide E2 puede causar daños en los organismos sujetos a consumo de alimento impregnado del esteroide, donde se encontraron anormalidades en el hígado y la presencia de gónadas intersexos en el esturión $A$. brevirostrum. Asimismo, Hendry et al. (2003) reportan deformidades físicas y crecimiento bajo en el lenguado de invierno Hippoglossus hippoglosus, cuando a las crías se les suministró alimento que contenía $10 \mathrm{mg} \mathrm{kg}^{-1}$ de $17 \beta$-estradiol por períodos de 45 días. De igual forma, Islinger et al. (2003) reportan cambios ultraestructurales en el hígado y testículos de machos del pez cebra Danio rerio expuestos a dosis de $85 \mathrm{mg} / \mathrm{L}$ de $17 \alpha$ - etinilestradiol por 21 días. Por último, es preciso resaltar la importancia en el balance entre andrógenos y estrógenos, el cual es esencial para el normal desarrollo sexual, para la función reproductiva, para el crecimiento normal y la fisiología de ambos sexos en los vertebrados. Tanto la expresión como la actividad de la enzima aromatasa mantienen este crítico balance de la producción de andrógenos y estrógenos (Conley y Walters, 1999). Algunos estudios muestran que la aromatasa, por el hecho de encontrarse al final de la ruta esteroidogénica, actúa como enzima clave en la producción de andrógenos $v s$. estrógenos y, por consiguiente, en el proceso de diferenciación sexual (Jeyasuria y
Place, 1998), lo cual ha sido observado en especies como el salmón real, Oncorhynchus tshawytscha, y el lenguado japonés, Paralychthys olivaceus.

\section{CONCLUSIONES}

El uso de nauplios y biomasa de Artemia spp. como vehículo del $17 \beta$-estradiol, además de la aplicación del andrógeno en el alimento balanceado para trucha en concentraciones de $50 \mathrm{mg} \mathrm{kg}^{-1}$ de medio o de alimento, respectivamente, realizando una estrategia de coalimentación, permiten una adecuada adaptación al consumo de alimento artificial, teniendo un efecto feminizante sobre las gónadas, incrementando considerablemente la proporción de hembras hasta en un $93 \%$ con respecto al grupo control con $100 \%$ de machos. En este aspecto, la mejor respuesta en la proporción de hembras se obtiene al alimentar a los juveniles por tiempos prolongados (21 a 42 días), observándose un efecto inverso, donde el crecimiento en los peces alimentados con $17 \beta$-estradiol por más días disminuye el peso y la talla de los peces, lo cual posiblemente es atribuido a un efecto adverso del esteroide, aunque esto no se manifiesta en relación con la supervivencia.

\section{AGRADECIMIENTOS}

Esta investigación fue financiada por la Universidad Juárez Autónoma de Tabasco (UJAT) a través del proyecto "Desarrollo del desove y larvicultivo del robalo blanco (Centropomus undecimalis) (Bloch, 1972)" con clave UJAT-2005CO1-42 y por The Aquaculture (CRSP) is funded in part by United States Agency for International a través del proyecto "Development of aquaculture techniques 
for indigenous species of Southern Mexico (Centropomus undecimalis): Sex determination and differentiation and effects of temperature". Twenty Fifth Annual Technical Report. Seedstock Development \& Availability 4 (12SDA4). Texas Cooperative Fish and Wildlife Research Unit es patrocinado por U.S. Geological Survey, Texas Tech University, Texas Parks and Wildlife, The Wildlife Management Institute, y U.S. Fish and Wildlife Service.

\section{BIBLIOGRAFÍA}

Aguilar, M., Coutiño, B. B. \& Salinas, R. P. (1996). Manual General de Técnicas Histológicas e Histoquímicas. México, D. F.: Las Prensas de Ciencias, Facultad de Ciencias.

Álvarez-Lajonchére, L. S. (2004). Cultivo de robalos: Potencialidades e resultados. Panorama Acuícola, 14(85), 15-21.

Caballero, V. (1996). Biología reproductiva del robalo Blanco (Centropomus undecimalis) en la zona suroeste del estado de Campeche. CRIP Cd. del Carmen. Informe Técnico del Instituto Nacional de la Pesca (inédito). Campeche, México: NAPESCA. Cd. del Carmen.

Chang, C. F. \& Lee, M. F. (1992, junio). Involvement of estradiol $b 17$ on the processes of natural and control sex reversal in protandrous black porgy (Acanthopagrus schlegeli). Ponencia presentada. Second International Symposium on Fish Endocrinology. St. Malo, France.

Chapman, P., Cross, F., Fish, W. \& Jones, K. (1982). Study 1: Artificial culture of snook. Final report for sportfish introduction projects. Florida, EE.UU.: Game and Fresh Water, Fish Commission.
Colombo, G. \& Grandi, G. (1990). Gonad sex differentiation of Anguilla anguilla by sex steroids. Int. Rev. Hydrobiol., 76, 763-773.

Conley, A. J. \& Walters, K. W. (1999). Aromatization. En E. Knobil \& J. D. Neill (Eds.), Encyclopedia of Reproduction. Vol. 1 (pp. 280-291). New York, EE.UU.: Academic Press.

Contreras-Sánchez, W. M., Márquez-Couturier, G., Feist, G., Hernández-Franyutti, A., Schreck, C. B. \& Giannico, G. (2003). Diversification of Aquacultural practices by incorporation of native species and implementation of alternative sex inversion techniques. Twenty-first Annual technical reports. Corvallis, Oregón, EE.UU.: Aquaculture CRSP.

Contreras-Sánchez, W. M., HernándezVidal, U., Hernández-Franyutti, A. A., Álvarez-González, C. A., Márquez-Couturier, G., Paramo-Delgadillo, S., Vidal-López, J. M., MoralesLara, G., De la Cruz-Rodríguez, M., Castro-Vasconcelos, C. C., BarabataDe la Cruz, J. L., Patiño, R., Pope, K. \& Huber, C. (2007). Development of aquaculture techniques for indigenous species of Southern México, Centropomus undecimalis: Sex determination and differentiation and effects of temperature. Seedstock Development \& Availability 4 (12SDA4). In K. Kosciuch (Ed.), Aquaculture Collaborative Research Support Program: Sustainable Aquaculture for a Secure Future (pp. 212-228). Oregon, EE.UU.: Aquaculture CRSP.

Devlin, R. H. \& Nagahama, Y. (2002). Sex determination and sex differentiation in fish: an overview of genetic, physiological, and environmental influences. Aquaculture, 208(3-4), 191-364. 
Flynn, S. R. \& Benfey, T. J. (2007). Effects of dietary estradiol-17 $\beta$ in juvenile shortnose sturgeon (Acipenser brevirostrum), Lesueur. Aquaculture, 270, 405-412.

Goudie, C. A., Redner, B. D., Simco, B. A. \& Davis, K. B. (1983). Feminization of channel catfish by oral administration of steroid sex hormones. Trans. Am. Fish. Soc., 112, 670-672.

Green, B. W., Veverica, K. L. \& Fitzpatrick, M. S. (1997). Fry and fingerling production. In H. S. Egna \& C. E. Boyd (Eds.), Dynamics of Pond Aquaculture (pp. 215243). New York, EE.UU.: CRC Press.

Grier, H. J. \& Taylor, R. G. (1998). Testicular maturation and regression in the common snook. J. Fish Biol., 53, 521-542.

Grier, H. J. (2000). Ovarian germinal epithelium and folliculogenesis in the common snook Centropomus undecimalis (Teleostei: Centropomidae). $J$. Morphol., 234, 265-281.

Happe, A. \& Zohar, Y. (1988) Self-fertilization in the protandrous hermaphrodite Sparus aurata: Development of technology. In Y. Zohar \& B. Brenton (Eds.), Reproduction in Fish - Basic and Applied Aspects in Endocrinology and Genetics (pp. 177-180). Italy, Paris: INRA Press.

Hendry, C. I., Martin-Robichaud, D. J. \& Benfey, T. J. (2003). Hormonal sex reversal of Atlantic halibut (Hippoglossus hippoglossus L.). Aquaculture, 219, 769-781.

Huber, C. G. (2007). Habitat preferences of juvenile common snook in the Lower Rio Grande, Texas. Tesis de maestría no publicada, Texas Tech University, Texas, EE.UU.

Hunter, G. A. \& Donaldson, E. M. (1983). Hormonal sex control and its application to fish culture. En W. S. Hoar, D. J.
Randall \& E. M. Donaldson (Eds.), Fish Physiology, Reproduction, Behavior and Fertility Control (pp. 223-303). New York, EE.UU.: Academic Press.

Islinger, M., Wilimsky, D., Völkl, A. \& Braunbeck, T. (2003). Effects of $17 \alpha-$ Ethynilestradiol on the expression of three estrogen-responsive genes and cellular ultrastructure of liver and testes in male zebrafish. Aquatic Tox., 62, 85-103.

Jeyasuria, P. \& Place, A. R. (1998). Embryonic brain-gonadal axis in temperature-dependent sex determination of reptiles: a role for $\mathrm{P} 450$ aromatase (CYP19). J. Exp. Zool., 281, 428-449.

Johnstone, R., Simpson, T. H. \& Youngson, A. F. (1978). Sex reversal in salmonid culture. Aquaculture, 13(2), 115-134.

Marshall, A. R. (1958). A survey of the snook fishery of Florida, with studies of the biology of the principal species, Centropomus undecimalis Bloch. Florida. State Bd. Conser. Tech. Ser., 22, 5-37.

Nakamura, M. (1984). Effects of estradiol$17 \beta$ on gonadal sex differentiation in two species of salmonids, the masu salmon, Oncorhynchus masou, and the chum salmon, O. keta. Aquaculture, 43(1-3), 83-90.

Pandian, T. J. \& Sheela, S. G. (1995). Hormonal induction of sex reversal in fish. Aquaculture, 138, 1-22.

Perera-García, M. A., Mendoza-Carranza, M., Contreras-Sánchez, W. M., HuertaOrtiz, M. \& Pérez Sánchez, E. (2011). Reproductive biology of common snook Centropomus undecimalis (Perciformes: Centropomidae) in two tropical habitats. Rev. Biol. Trop., 59(2), 669-681.

Peters, K. M., Matheson, R. E. Jr. \& Taylor, R. G. (1998). Reproduction and early life history of common snook, 
Centropomus undecimalis (Bloch) in Florida. Bull. Mar. Sci., 62(2), 509-529. Phelps, R. P., Lovshin, L. L. \& Green, B. W. (1996). Sex Reversal of Tilapia: $17 \alpha$-Methyltestosterone Dose Rate by Environment and Efficacy of Bull Testes. Progress Report, Honduras Special Study 1. Oregon, EE.UU.: Pond Dynamics/Aquaculture Collaborative Research Support Program.

Piferrer, F. (2001). Endocrine sex control strategies for the feminization of teleost fish. Aquaculture, 197(1-4), 229-281.

Rivas, L. R. (1986). Systematic review of the perciform fishes of the genus Centropomus. Copeia, 3, 579-611.

Sánchez, Z. A., Rosas, V. C., Durruty, L. C. V. \& Suárez-Bautista, J. (2002). Reproducción en cautiverio de robalo, una necesidad inaplazable en el sureste mexicano. Panorama Acuícola Mag., 6, 24-25.

StatPoint, Inc. (2006). STATGRAPHICS (Data analysis software system), Version 5.1. www.statgraphics.com. Warrenton, EE.UU.: StatPoint, Inc.

Stewart, A. B., Spicer, A. V., Inskeep, E. K. \& Dailey, R. A. (2001). Steroid hormone enrichment of Artemia nauplii. Aquaculture, 202(1-2), 177-181.

Strüssmann, C. A., Takashima, F. \& Toda, K. (1996). Sex differentiation and hormonal feminization in pejerrey Odontesthes bonariensis. Aquaculture, 139(1-2), 31-45.

Taylor, R. G., Whittington, J. A., Grier, H. J. \& Crabtree, R. E. (2000). Age, growth, maturation and protandric sex reversal in common snook, Centropomus undecimalis, from the east and west coasts of Florida. Fish Bull., 98, 612-624.

Tringali, M. D. \& Bert, T. M. (1996). The genetic stock structure in common snook (Centropomus undecimalis). Can. J. Fish. Aquatic Sci., 53(5), 974-984.

Tucker, J. W. Jr. (1987). Snook and tarpon snook culture and preliminary evaluation for commercial farming. Prog. Fish. Cult., 49, 49-57.

Tucker, J. W. Jr. (2003). Snook culture. World Aquac. Soc. Mag., 34(4), 42-46.

Vázquez, G. A. (2008). Evaluación del efecto feminizante de la administración del esteroide 17ß-estradiol en juveniles del pejelagarto (Atractosteus tropicus). Tesis de Licenciatura no publicada. División Académica de Ciencias Biológicas, Universidad Juárez Autónoma de Tabasco, México.

Vidal-López, J. M., Álvarez-González, C. A., Contreras-Sánchez, W. M. \& Hernández-Vidal, U. (2009). Masculinización del cíclido nativo Tenhuayaca, Petenia splendida (Günther, 1862), usando nauplios de Artemia como vehículo del esteroide 17- $\alpha$ metiltestosterona. Hidrobiológica, 19(3), 211-216.

Yamamoto, T. O. (1969). Sex differentiation. En W. S. Hoard \& D. J. Randall (Eds.), Fish Physiology (pp. 117-175). New York, EE.UU.: Academic Press.

Zarza, E. A., Berruecos, J. M., Vázquez, C. \& Álvarez, P. (2006). Cultivo experimental de robalo Centropomus undecimalis (Bloch, 1792) y Chucumite Centropomus parallelus (Poey, 1860) (Perciformes: Centropomidae) en agua dulce en un estanque de concreto en Alvarado, Veracruz, México. Vet. Méx., 37(3), 327-333. 